\title{
La cuestión de la laicidad en Augusto Del Noce
}

\section{Paolo Armellini*}

Sapienza Università di Roma paolo.armellini@uniroma1.it
Revista Cultura Económica

Año XXXIX $\bullet \mathrm{N}^{\circ} 102$

Diciembre 2021: 85-100

https://doi.org/10.46553/cecon.39.102.2021.p85-100

Resumen: La reflexión de Augusto Del Noce sobre la modernidad abarca desde el análisis de los fenómenos revolucionarios hasta la recuperación de los valores tradicionales en las sociedades secularizadas. Desde este punto de vista, pertenece a la corriente de pensamiento político que en la época contemporánea ha intentado repensar la categoría de laicidad para salir no solo del laicismo, que no admite ninguna relación entre la religión y la esfera pública, sino también de una utopía arqueológica que pretende restablecer un estrecho vínculo entre los valores religiosos y las estructuras institucionales de la política. La política actual debe superar esta antinomia estéril para establecer una sana relación entre las dimensiones ético-religiosas de la democracia y su ineludible autonomía.

Palabras clave: Laicado; Modernidad; Revolución; Religión; Tradición

\section{The issue of secularity in Augusto Del Noce}

Abstract: Augusto Del Noce's reflections on modernity range from the analysis of revolutionary phenomena to the recovery of traditional values in secularized societies. From this point of view, he belongs to the current of political thought that in contemporary times has attempted to rethink the category of secularisation in order to move away not only from secularism, which does not admit any relationship between religion and the public sphere, but also from an archeological utopia that seeks to reestablish a close link between religious values and the institutional structures of politics. Today's politics must overcome this sterile antinomy in order to establish a healthy relationship between the ethical-religious dimensions of democracy and its inescapable autonomy.

Keywords: Laity; Modernity; Revolution; Religion; Tradition

* Recibido: 02/12/2021 - Aprobado: 16/12/2021 


\section{Introducción}

Del Noce ha sido descrito a menudo como un filósofo aislado e incomprendido, especialmente por los católicos. Nunca ha estado satisfecho con el hecho de que una de las sociedades más corruptas y descristianizadas de Occidente haya crecido a la sombra de un partido de inspiración cristiana. Sin embargo, esto es comprensible a la luz del hecho de que nunca quiso confundir su papel de intelectual, especialmente como filósofo católico, con el de consejero del príncipe que estudia estrategias para lograr resultados. Esto lo ha mantenido siempre alejado de la praxis política que ha mostrado la Democracia Cristiana (en adelante, DC). Más bien pensó en volcar su propia experiencia cultural en la acción política concreta, manteniendo su propia autonomía de criterio. En efecto, si la de Del Noce no era simplemente una fe contemplativa, sino también activamente militante y capaz de descender a la praxis por su convicción del peso decisivo que la cultura tiene en el orden político, pensaba sin embargo en su propio papel no como una elaboración de datos a combinar para alcanzar objetivos inmediatos, sino como una posición crítica que enuncia tesis relativas a los juicios de valor inherentes a los fines últimos y mediatos (Mondin, 1990: 58-62).

El pensamiento de Del Noce fue analizado por el difunto Claudio Napoleoni (Napoleoni, 1990; 1992) quien se preguntó si era posible salir de la sociedad opulenta por una vía puramente política. Napoleoni, en comparación con la posición de Franco Rodano, que no vio otras razones para el funcionamiento político que las propias de la política, apoyadas únicamente en la referencia a una moral natural que valora los ideales de libertad e igualdad, afirma que la crítica teológica a la posición que busca en el renacimiento del pensamiento jesuítico del siglo XVII la garantía de una plena autonomía y bondad de lo finito respecto a lo sobrenatural la ofreció el propio Del Noce al observar que el separatismo molinista es una de las premisas de la irreligión occidental. Ciertamente habría que investigar hasta qué punto la valoración que Rodano hace del molinismo depende de una interpretación errónea de Santo Tomás, pero lo cierto es que la afirmación de la autosuficiencia absoluta de la naturaleza pura del hombre lleva también, según Napoleoni, a la superfluidad de cualquier referencia a la trascendencia. ¿La exclusión de la trascendencia del horizonte de la modernidad no sería la raíz de toda la violencia que en ella se produjo finalmente? ¿No sería conveniente, entonces, volver a las tesis del Concilio de Calcedonia, que tanto insistieron en la proximidad de Dios y del hombre como fuente de auténtica fraternidad? 


\section{Secularización y revolución}

El pensamiento de Del Noce se mueve al nivel de estas cuestiones, reflexionando sobre todo en el papel político de los católicos en relación con la crisis de la idea moderna de laicidad, pensada como una categoría filosófica independiente de cualquier referencia religiosa. De hecho, si en el período de De Gasperi un acuerdo sobre los principios éticos de los laicos y los católicos era todavía un presupuesto indiscutible, con la revolución neoburguesa en Italia se produjo la máxima expansión, en las costumbres y la conciencia colectiva italiana, del programa gobettiano y gramsciano de modernización del país (Del Noce, 1978; 1998, en lo sucesivo denominado CL). Por ello, nos parece que las reflexiones de Augusto Del Noce en el período comprendido entre 1974 y 1989 (años en los que Del Noce se acercó a Comunione e liberazione y al Movimento popolare) están marcadas por la profundización del tema maritainiano de la laicidad (Possenti, 1992).

Aunque en Europa, tras el periodo de totalitarismo comunista, fascista y nazi, existía una mayoría de partidos católicos que insinuaban un renacimiento de los principios y un nuevo cristianismo, el clima posterior cambió catastróficamente. La DC, un partido de inspiración cristiana que se inspiró en la lección filosófica y en la visión social de León XIII, se encontró desempeñando el papel de guardián democrático del proceso de secularización en un momento en el que la democracia laica aún no era capaz de asumirlo, dejando que la DC ocupara el lugar. En su gestión pragmática del poder como control anticomunista, la DC dejó de lado las cuestiones culturales para evitar tensiones. Sin embargo, al hacerlo, permitió pasivamente que la relación particularmente delicada entre cultura y política en Italia difundiera el juicio de que la historia contemporánea podía explicarse por la confrontación entre la línea progresista, anticlerical y atea, y la línea reaccionaria de la que el fascismo era la expresión necesaria. La primera habría sido la respuesta adecuada en sí misma a las posturas integristas de la segunda, de modo que, si esta hubiera sido erradicada algún día, el laicismo y el comunismo habrían perdido su rostro anticlerical y ateo. Este juicio histórico se habría colado subrepticiamente incluso entre los católicos, cuyo primer adversario habría estado entre ellos mismos: el integrismo. Esto se debió a la consolidada desconfianza del pragmatismo político cristiano hacia las ideologías míticas y las filosofías modernas de la historia: la religión de la libertad para los liberales, la herencia secular del Risorgimento para los republicanos, la modernización para los socialdemócratas y la revolución para los comunistas. La DC, pensando que 
la religión solo se refería a la salvación del alma, dejó el cuidado de las cosas temporales a la política, sancionando de hecho la separación entre religión y política, que en principio se llamaría "responsabilidad autónoma del laico" (Tamassia, 1992). Por otro lado, para el pragmatismo político de los "demócratas" de la DC la democracia constituía "un régimen de plena autonomía de principio, de separatismo de hecho, entre la religión y la política; y los católicos podrían ser sus líderes, debido al carácter trascendente de la religión" (Del Noce, CL: 68).

Pero la fe reducida a un asunto puramente privado era funcional a una sociedad permisiva, falsamente tolerante incluso para los laicos más perspicaces, porque la secularización no es simplemente una novedad necesaria de la que hay que tomar nota, sino la caída no solo de la fe sino también de los valores, ante los que hay que ejercer la libertad de conciencia. Por ello, la inspiración cristiana del partido de los católicos democráticos no puede disociarse, según Del Noce, de la integridad de una filosofía católica de la historia, sin la cual el anti-ideologismo hacia el que se había inclinado la DC correría continuamente el riesgo de caer en el contraste con la afirmación de Juan Pablo II de que la actividad social y política del hombre debe hacer más humana la organización social y no solo técnicamente más eficiente (Del Noce, 1983b).

La crítica al catolicismo realizada en sentido historicista se ha centrado en el hecho de que el proceso de descristianización se ha producido sin ningún odio aparente hacia el cristianismo, en la convicción, expresada por Gramsci, de que el catolicismo reducido a mera regla de vida es la crítica más rigurosa de sí mismo y, por tanto, está destinado al suicidio por ser inaplicable en todos los actos de la vida. El cristianismo era verdadero, pero hoy ya no lo sería: esta es la condena de la que serían víctimas los católicos. Su fe parece destinada a ser vivida en un doble juego, según una actitud separatista, por la que se limita a la esfera privada, mientras que en la esfera pública los cristianos deben perder la cara como sujetos activos en la historia y las instituciones.

\section{La interpretación católica de la laicidad}

¿De qué interpretación de la historia depende este separatismo? Para Del Noce, sus formas podrían reducirse a tres: la utopía arqueológica, que eleva el ideal histórico de la Edad Media a un modelo válido para siempre según su idealización romántica, lleva al cristiano a vivir en la modernidad en una especie de ocultamiento, pero la crítica histórica le lleva la delantera. La 
utopía del futuro es exactamente la inversa de la anterior porque el cristianismo significa el crecimiento de la humanidad, por lo que la Iglesia ya no debe ejercer una función de sustitución hacia las tareas históricas concretas del hombre. Ahora vive en la época de su autosuficiencia, siendo los tiempos modernos los siglos del cumplimiento del cristianismo, porque la Redención ha puesto la historia en manos del hombre. Esta posición da lugar a la tesis de que la fe no tiene nada que decir sobre las opciones temporales y políticas. Y el llamado proceso de secularización, que la Iglesia no habría reconocido, generó por contraste el laicismo como respuesta al malentendido por el que el catolicismo "integrista" pensaba que la sacralización de la política y la religión eran inseparables, justificando el orden establecido y, como contrapartida, la condena religiosa del mundo moderno que generó el enfrentamiento entre reaccionarios y progresistas. Un tercer tipo de separatismo deriva del pragmatismo de la desideologización de la política a través de un silencio sobre los objetivos últimos en nombre de la solución concreta a la que aspiran los problemas empíricos (Del Noce, 1983b).

¿Cuál fue la interpretación que prevaleció entre los católicos en la posguerra? Una interpretación muy sugerente en este caso es la interpretación innociana de la historia de la Universidad Católica, en la transición de la filosofía de Olgiati y Gemelli al dossettismo. Desde la actitud profascista inicial, dictada por el hecho de que el fascismo, surgido de matrices no católicas tras la Primera Guerra Mundial (comúnmente entendida como una guerra contra los restos medievales del Imperio de los Habsburgo y la Iglesia), parecía luchar contra los mismos enemigos de la Iglesia (la masonería, el liberalismo). Una vez derrotados, se podía pensar en una posterior restauración católica, lo que llevó a la conversión antifascista de jóvenes católicos como Dossetti y Lazzati que, alejados de la política, pensaron que era su deber dedicarse a ella debido a la nueva amenaza global del nazifascismo. De ahí su compromiso con la Resistencia, interpretada como un fenómeno histórico unitario que transformaría las fuerzas que habían participado en ella. Los católicos abandonarían las tentaciones nostálgicas y reaccionarias y se adherirían plenamente a la democracia, mientras que los comunistas -se esperaba- abandonarían su superestructura atea. Dossetti también pensaba que el gran error de la Iglesia en la época moderna había sido ponerse del lado de la reacción, un defecto que se correspondía con un defecto teológico que se remontaba al Concilio de Trento y a la Contrarreforma. En 1945-48, el frente unitario antifascista se rompe y se forma el bando anticomunista en torno a la DC de De Gasperi, que según Dossetti, no pudo recuperar lo positivo del comunismo. Además, su posición 
fundamentalista de estricta unión entre religión y política contrastaba con la de De Gasperi, que buscaba una distinción de términos, manteniendo la inspiración cristiana. Esto explica su abandono de la política activa por una búsqueda teológica que hubiera conducido a una verdadera reforma de la Iglesia (Del Noce, 1985; 1988g; 1987b).

Su fracaso político fue paralelo al del Partido de Acción, nacido del intervencionismo democrático-revolucionario de la Primera Guerra Mundial, que tras su derrota política pasó de la política activa a la cultura. Su objetivo era promover una hegemonía cultural en la república de las letras con el programa de secularización de la escena política contra los vicios estratificados en la historia italiana desde la Contrarreforma hasta el fascismo, visto como el mal radical del siglo XX. El Partito d'Azione creó así el mito antifascista, representándose a sí mismo como la síntesis de las fuerzas modernas que habían combatido el fascismo y el nazismo y ejerciendo, tras la decadencia comunista, esa cultura democráticoprogresista que redescubrió la disposición espiritual de la Ilustración para interpretar la historia en términos de progreso y reacción, apreciando el poder de negatividad de la tradición católica. Si los católicos antifascistas aceptaban también la tesis de que el mal moderno provenía del integrismo, que tiene sus raíces remotas en la Contrarreforma, era natural que el enemigo fuera ese catolicismo que todavía pensaba en una ciudad cristiana ideal:

El llamado integrismo, definido como la alianza del catolicismo con un orden histórico determinado, que lo instrumentalizaría en su defensa. Fue a través de esta antítesis de integrismo y progresismo que las valoraciones de la Ilustración pudieron penetrar en la cultura de los católicos (Del Noce, 1988h; ahora en CL: 158).

La política de los católicos democráticos estaba así informada por la idea de que la democracia estaba conectada con la afirmación del laicismo, entendido como la neutralidad de la política, completamente separada de la fe por la trascendencia absoluta de Dios (Del Noce, 1988b; 1988j; ahora en CL: 275-280; 161-169).

En un primer momento, el ataque a la cultura marxista se desarrolló a través del resurgimiento del gramscismo, heredero de la cultura de Croce. Después de los años sesenta le sucedió la cultura laico-burguesa, que sin embargo acepta la crítica marxista de todo absolutismo y eternidad de los valores. El tipo cristiano-burgués, que afirma el cristianismo sin trascendencia religiosa, pero salva la moral católica, es sustituido por el tipo 
secular post-marxista, que abandona los aspectos mesiánicos del marxismo para conservar los no cristianos. Considera agotada la idea de Dios y su función en la historia, y muerto el cristianismo en su forma tradicional, según ese programa gramsciano de 1916 según el cual el socialismo es la religión que debe sustituir al cristianismo.

Ahora bien, la auto-confutación del marxismo ha llevado a su descomposición, con implicaciones diferentes en Oriente y en Occidente, que sin embargo tienen la misma raíz. El resultado ha sido la complementariedad entre la heterogénesis de fines a la que ha estado sometida la revolución comunista y la aparición en Occidente de la mentalidad consumista, que es un fenómeno cultural antes que económico, y que se expresa en forma de secularización en el modo de tener. Ambos tienen en común el talante materialista y la negación de los valores. Si Marx tiene el mérito de haber intentado romper las cadenas de lo económico, su fracaso ha determinado, también y sobre todo en Occidente, la nueva forma de alienación que es la mercantilización recíproca de los sujetos existentes en la sociedad opulenta (Del Noce, 1983c). Del Noce expone su tesis de la siguiente manera: "La historia contemporánea, entendida en el sentido más amplio de la historia del mundo, solo puede entenderse como la historia de la realización del marxismo, de su éxito y al mismo tiempo de su fracaso" (Del Noce, 1983c; ahora en CL: 158), sin poder separar lo primero de lo segundo.

En el análisis de Del Noce, este resultado se aclara si se examina seriamente el fenómeno de la revolución comunista en su estrecha relación con los escritos de Marx. A Del Noce le llamó la atención una frase de Croce sobre el sentido de la responsabilidad: se es responsable porque la historia nos hace serlo, en relación con una tarea práctica. Pues bien, según Del Noce, esta posición representa lo demoníaco del historicismo para la tesis de la responsabilidad "atribuida". Croce, en los últimos años de su vida, con la explosión del totalitarismo, tomó conciencia de este aspecto demoníaco del inmanentismo ético, que con Lenin pasó por completo a la revolución comunista. $\mathrm{Si}$, en efecto, no es el individuo el responsable de sus propios actos en relación con un orden suprapersonal de valores, es "hecho así por otros, y estos otros son los que tienen el mayor poder, ninguna columna del historicismo puede escapar a la ley del más fuerte" (Del Noce, 1988f, ahora en CL: 203). El leninismo, desarrollando un motivo implícito en Marx, afirma la idea de la objetividad de la culpa, por la que se es culpable, más allá de cualquier intención moral, por la significación que nuestras acciones asumen objetivamente en la historia. Será el partido que juzgue lo que sirve a la 
revolución, es decir, como mediador de la transición a una nueva realidad, juzga como moral solo lo que sirve a la política revolucionaria. La ética está, pues, en el marxismo-leninismo completamente subordinada a la política (Vasale, 1995).

\section{La secularización en Occidente}

¿Cómo pudo el marxismo llegar a esta posición coherente? La tesis que enuncia Del Noce parte de la consideración de que la historia contemporánea es la historia de la realización del marxismo, en el sentido de que ha triunfado y fracasado al mismo tiempo. El marxismo debe ser considerado en primer lugar en su primer momento filosófico, que es la crítica de la religión que tuvo lugar en la izquierda hegeliana, por lo que se presenta bajo el aspecto de contrarreligión o religión laica para la difusión del ateísmo más radical como rechazo de toda dependencia. No se presenta como una comprensión de una totalidad ya realizada, sino como la realización de una totalidad, que en la religión toma el aspecto de la redención. Cualquier inversión de la misma es imposible y acaba más bien en descomposición, dando lugar a la más gigantesca heterogénesis de fines jamás vista en la historia. De hecho, el marxismo ha sido la mayor síntesis de contrarios de la historia del pensamiento, porque combina el colmo de la utopía - para la que la nueva sociedad, imposible de representar, es totalmente otra y sin analogía con la realidad actual-con el colmo del realismo político para la tesis de la inclusión de la ética en la política. La intención esencial del pensamiento marxiano es el rechazo de toda dependencia y la extinción de la religión por la que es Dios el Primer Señor. El pensamiento revolucionario ve un vínculo necesario entre la extinción de la religión y la del Estado, que es su base mundana. ¿Quién será el sujeto de la transición a la nueva humanidad? No el proletariado, que no puede ser el guía sino solo el mediador de este pasaje. Lenin en ¿Qué hacer? (1903), expone que la conciencia política de clase solo puede ser llevada al proletariado desde fuera. El proletariado se convierte así en la herramienta de los nuevos gnósticos que no son intelectuales ordinarios, sino los que conocen el curso de la historia en su totalidad y tienen las claves del futuro.

Consideremos ahora el fracaso de este intento. En Marx, la desaparición de la religión debía coincidir con la recuperación por parte del hombre de aquellos poderes que había enajenado para proyectarlos en el curso de la historia en Dios. En Occidente, el marxismo ha tenido la función de corroer la fe en lo absoluto de los valores. La producción de nihilismo fue su resultado porque fue incapaz de sustituir los viejos valores menguantes por 
otros nuevos a través de la fe revolucionaria. El comunismo se mostró así incapaz de subvertir la dominación burguesa, que pudo adoptar la nueva forma de una sociedad tecnocrática y opulenta, contra la que el marxismo no ofrece armas contundentes. En Occidente tenemos el pleno despliegue del pluralismo libertario, que rechaza la idea de una moral universal y reduce el discurso ético-político al de las técnicas sociológicas de convivencia. El comunismo y la democracia occidental no son, pues, tesis y antítesis que puedan unificarse en una nueva síntesis, sino elementos de la descomposición del marxismo, al que se ha llegado contradiciendo sus premisas.

En este sentido, la oposición a Norberto Bobbio es aquí más fuerte:

La historia contemporánea ofrece la ventaja de la plena racionalidad. Hace poco leí -dice Del Noce- una sentencia de Bobbio, según la cual la realidad contemporánea desmiente la suposición de que la historia de las ideas y la historia de los hechos discurren por la misma vía, para demostrar que no hay nada menos racional que la creencia en la racionalidad de la historia; y no es una broma, porque esta sentencia es el punto de llegada de una de las direcciones predominantes de la cultura filosófico-política italiana, la que partió de Gobetti y se inspiró en Cattaneo, es decir, el socialismo liberal. Para Bobbio, el mundo contemporáneo está marcado por la lucha modernidad-igualdad-democracia, tres ideas unidas para él, contra el irracionalismo, por la antítesis de Rousseau y Nietzsche. Para mí, sin embargo, para comprender su historia, es decir, la que va desde la Primera Guerra Mundial y la Revolución de Octubre hasta nuestros días, hay que dar prioridad a la causalidad ideal, tanto como al momento filosófico y religioso (Del Noce, 1990; ahora en CL: 254-255).

Si todo esto es cierto, el marxismo

se presenta como el punto más alto de la modernidad [...] sin embargo, también representa la crisis de la modernidad, una crisis que no puede ser superada porque el marxismo está sujeto a la descomposición, pero no es susceptible de revertirse, y esta crisis se puede ver correr en la misma pista de la historia de las ideas y la historia de los hechos que Bobbio niega (Del Noce, 1990; ahora en CL: 256-257).

Con respecto a este panorama, que podría llevar al pesimismo, a Del Noce nunca le faltó la energía intelectual para buscar una nueva política 
cultural necesaria para combatir la creciente descristianización en Italia. El análisis de Del Noce pretendía cuestionar una interpretación del papel de los católicos en la política, según la cual la DC dejaba de representar a los creyentes para convertirse en el partido que conducía a los católicos a la democracia. De este modo, la inspiración cristiana solo se entendía como el fundamento de la máxima secularidad de la política, que se deriva del dualismo entre lo espiritual y lo temporal, basado en el supuesto de la trascendencia divina. La secularización desde este punto de vista sería la plena madurez de la humanidad frente a la inmadurez de los medievales que delegaban en la Iglesia todo tipo de sustituciones. Siguiendo esta línea interpretativa, los católicos, más que buscar su propia identidad en el marco democrático, deberían salir de la situación de minoría psicológica en relación con el mundo moderno para adherirse plenamente a la conciencia democrática caracterizada por el sufragio universal, la libertad política parlamentaria, la elevación cultural del pueblo independientemente de las creencias religiosas. Si las creencias religiosas siguen teniendo una función, es la de ser una fuerza vitalizadora con respecto a una tarea política, exclusivamente secular en su naturaleza, que sin embargo corre el riesgo de confinar la vida religiosa a la esfera privada sin que tenga ningún impacto en la historia de la vida pública. Ahora bien, incluso la mejor tradición del personalismo (piénsese en el último Ricoeur) ha percibido una especie de antinomia radical y latente entre las categorías de comunidad y sociedad, por la que en la primera se corre el riesgo de una infravaloración sistemática de las instituciones y el rechazo paralelo de la sociedad, con la consiguiente privatización de la vida familiar, la evasión de los deberes civiles y la huida de la política. La proximidad que concibe esta forma degenerativa de personalismo es solo la de la relación cara a cara, en la relación de amistad y de amor, y condena como anónima y sin rostro la relación que, a través de las instituciones y la vida pública, el hombre pretende establecer con "todos" (Ricoeur, 1993). Puede decirse que también Del Noce, al involucrarse directamente en la política con su candidatura y elección al Senado, sintió la necesidad de disolver esta antinomia ampliando la idea de amistad de lo interpersonal a lo civil. De hecho, siempre se ha mostrado insatisfecho con el carácter monástico de un cierto personalismo que tiende a yuxtaponer a Marx y Kierkegaard.

Pero también quiso distinguir el amor al prójimo propio del cristianismo, que tiene sus implicancias sociales y políticas, del humanitarismo burgués, que sustituye la caridad por la filantropía y la esperanza por la satisfacción. El amor cristiano se basa en la idea de que Dios 
no solo es creador, sino que lo es por medio del amor. La moral que se desprende de ella, centrada en lo divino en el hombre, está inextricablemente ligada a la visión religiosa del mundo. En cambio, ¿qué se pide hoy a los católicos sino la reducción del cristianismo a una ética social, separada, sin embargo, de la metafísica y de la teología, y por tanto juzgada capaz por sí misma de alcanzar la universalidad y la fundación de una sociedad justa? Con respecto a esta moral, el católico puede añadir una esperanza de otro mundo que desempeñe un papel vitalizador en la acción práctica. Pero la condición para que el católico se adhiera a esa moral universal es que su fe solo se yuxtaponga a la relación entre ética y política. La moral ecuménica humanitaria también puede expresarse en el lenguaje del catolicismo, pero este no debe convertirse en una fuente de división. En el ecumenismo humanitario que profesa la masonería, el rito católico solo se tolera si abandona sus características integristas (Del Noce, 1988b).

En el camino de este proceso, gana terreno la idea de que la plena autonomía y autosuficiencia del hombre lo hacen capaz de fundar una buena civilización al margen de cualquier religión. El programa de Bayle de una ciudad de ateos sería así posible no tanto en el sentido de una república que incluyera el ateísmo en su constitución, sino en el sentido de que la palabra de Dios habría perdido su importancia para su fundación.

\section{El destino de la modernidad y la laicidad}

De ahí la recuperación de la idea de Maritain de una nueva cristiandad que Del Noce pretende rehabilitar para oponerse a una sociedad completamente agnóstica, para la que los valores son objeto de consumo y se reducen a simple valor de cambio. Aquí debemos registrar una paradoja. El propio Del Noce, que se deja cuestionar seriamente por las tesis de Gramsci sobre el suicidio del catolicismo entendido solo como simple norma de vida, es el que aparece más vinculado entre los autores católicos a esa idea del cristianismo que otros han dado por concluida en la modernidad. Del Noce siempre ha estado convencido de que el cristianismo no está superado históricamente en la modernidad según el decreto de las filosofías de la historia no católicas (hegeliana, marxista y positivista). Se puede salvar de sus resultados nihilistas si se reconoce que el verdadero moderno es el que descubre los propios límites de la modernidad, es decir, es problematicidad. Lo que hay que cuestionar es su sentido axiológico, que Del Noce comparte con el movimiento de Comunión y Liberación, nacido del magisterio de don Giussani que, en su método de comunicación de la fe, ha insistido siempre en que el acontecimiento cristiano tiene la posibilidad de hablar a todo hombre 
a partir de las condiciones ambientales de la vida, siempre que el hombre recupere su percepción originaria de dependencia de algo superior a él y reconozca que no puede darse a sí mismo la salvación. En el plano filosófico, esto coincide con la idea de Del Noce de que se puede hablar de Cristo en el presente si se elimina la raíz del racionalismo, que consiste en el rechazo no probado del dogma del pecado original, sin el cual no se puede entender la necesidad de la Redención (Del Noce, 1986b; 1986c; 1988d).

Por eso, Del Noce siempre cultivó la esperanza de un cristianismo capaz de influir en la historia institucional y en la sociedad, para contribuir en el presente a la formación de una nueva cultura en contraste con la del egocentrismo consumista. La nueva Kulturkampf, promovida por laicistas y marxistas contra el catolicismo y a favor de la indiferencia religiosa, parte de la vieja convicción de Croce de que el ideal liberal es claramente negado por el catolicismo y de la intención de que una nueva Ilustración, como síntesis de socialismo y liberalismo, solo puede partir de la idea de un contraste entre la moderna religión de la libertad y la conservadora y reaccionaria católica (Del Noce, 1987a).

Ante semejante adversario, Del Noce juzgó insuficiente la cultura del partido de inspiración cristiana. A menudo ha sucumbido a un pragmatismo desprovisto de motivaciones ideales, con una relativa pérdida de estilo cristiano. Una nueva política cultural de los católicos no debe confundir fe religiosa y política, pero no puede olvidar que el catolicismo no es una doctrina de espiritualidad separada de la vida y alejada del destino de la humanidad. La laicidad, por tanto, no puede significar la negación de la estructura esencial de encarnación del cristianismo. Maritain distingue el cristianismo de la idea de cristiandad subrayando que la verdad religiosa integral es la de la Iglesia católica, mientras que las diferentes cristiandades son aquellas civilizaciones cristianas que en la estructura de su régimen temporal común llevan en diversos grados la huella de la concepción cristiana de la vida (Maritain, 1969). Los que se han puesto del lado de la "opción religiosa" parecen creer en la idea de que la era del cristianismo ha terminado definitivamente y que la civilización podría organizarse y prosperar incluso sin referencia a la religión. Los católicos deberían así limitarse a contribuir a la promoción de los valores que la civilización secular afirma sin referencia religiosa a la ciudad, pero su objetivo último no sería ya la acción política que informa el régimen temporal de la concepción cristiana de la vida. La eliminación de la idea de cristiandad es, pues, directamente proporcional a la 
extensión arbitraria del término "autonomía" ligada a los malentendidos de la secularización en relación al catolicismo reaccionario.

Para Del Noce, la laicidad política de los católicos significa, en cambio, que el tratamiento de las cuestiones temporales debe mantener el sentido de la conexión de lo temporal con lo eterno, porque el cristianismo, que es ciertamente una verdad metahistórica, no es solo una fe en una realidad suprasensible, sino que determina el sentido y da orientación de la existencia. Esto no significa el carácter confesional de la política. Del Noce subrayó la diferencia entre su concepción de la laicidad tanto de la modernista, que contemplaba la función vitalizadora de la fe, como de la separatista, que resolvía la tarea del partido cristiano en la incorporación de los católicos a la democracia. Del Noce, en cambio, afirma la idea de una laicidad "católica", por la que el cristiano que actúa en el ámbito temporal no puede olvidar nunca que es tal, pero, lejos de pretender convertir a los demás por medios temporales, tiene como objetivo establecer las mejores condiciones para dicha conversión, ordenando la actividad política a este proceso.

La relación entre religión y política adquiere especial importancia en el discurso delnoceano, porque ve en el cristianismo una desmitificación de la sacralidad superior del Estado y, por tanto, la condición para la libertad de la política. De hecho, si el Estado no es portador de una autoridad religiosa, para su fundamento ético debe remitirse a otra comunidad. Para Del Noce se trata de la Iglesia, que, basada en la pertenencia voluntaria, solo puede imponer sanciones espirituales, pero no civiles. Esto demuestra lo ajenas que son a Del Noce las tentaciones medievalistas, porque allí donde la Iglesia se convierte en Estado -repite con Ratzinger- se pierde la libertad, que es un valor inalienable en la medida en que es la condición a partir de la cual se pueden reconocer los valores. Más bien, el peligro del que hay que protegerse son las nuevas formas de totalitarismo. El sistema totalitario contemporáneo no admite la dualidad Iglesia-Estado y tiende a reducir la fe a un mero hecho interior. La privatización de la religión, que es el fenómeno de los últimos cuarenta años, tiene como consecuencia la neutralidad de la democracia con respecto a los valores, reducida a reglas de juego que no son moralmente vinculantes. Para que la democracia no se corrompa y se convierta en anarquía o totalitarismo, hay que revisar la idea de un Estado que se presenta como autosuficiente respecto a la religión y en el que se aplica una tolerancia represiva que solo la admite como opinión privada (Del Noce, 1987a).

La novedad cristiana para Del Noce seguía siendo la alternativa a esos falsos avances de la historia que hacen del reino de Dios y sus versiones 
seculares un mero producto político, con la sustitución de la política por la religión para la liberación del hombre. La primacía universal de la política, que restablece la idea pagana de la divinidad del Estado, solo puede ser contrarrestada por una nueva relación entre la religión y la política, no separadas sino unidas, aunque sean distintas. La naturaleza humana solo puede respetarse plenamente si se reconoce que la persona está impulsada por un deseo de infinito, que no puede satisfacerse en esta tierra, pero que debe ordenar su vida individual y social. La verdadera democracia resulta ser ese régimen en el que todos pueden ser considerados siempre como un fin y nunca como un medio, y cuyo poder es la voluntad de servir a la criatura de Dios en la evolución dinámica de su deseo de infinito, impidiendo que se haga añicos y se reduzca a muchos pequeños deseos. La política pura, en cambio, pretende gobernar estos deseos satisfaciéndolos mediante el sometimiento consensuado de las conciencias heterodirigidas. Es la realización completa del totalitarismo como destrucción de la memoria histórica, que se lleva a cabo pasando de la opresión física y el exterminio de los cuerpos a la opresión moral y el exterminio de la inteligencia. Un individuo desarraigado del pasado y reducido así a una mens momentanea, incapaz de orientarse en el futuro, es en cambio el peligro que Del Noce denunció con firmeza, combatiendo la homologación de las masas al margen de cualquier elitismo aristocrático (Del Noce, 1988g; ahora en CL, 117-121).

\section{Referencias Bibliográficas}

Armellini, P. (1999). Razionalità e storia in Augusto Del Noce. Roma: Aracne.

Armellini, P. (2017). Le avventure della modernità in Augusto Del Noce. Roma: Nuova Cultura.

Castellano, D. (ed.) (1992). Augusto Doce. Il pensiero filosofico, Nápoles: ESI.

Del Noce, A. (1959). "Il Maritainismo 1935 e maritainismo 1959". L'Ordine civile, I, n. 4, 10 agosto.

Del Noce, A. (1973). "L'unità del pensiero di Maritain”. L'Europa, VII, n. 7, 30 aprile, pp. 77-86.

Del Noce, A. (1976). "Maritain e l'antimodernismo cattolico". Azione sociale cristiana, III, n. 2, febbraio.

Del Noce, A. (1978a). Il suicidio della rivoluzione. Milano: Rusconi.

Del Noce, A. (1978b). "La scelta di un maestro". L'Osservatore romano, 20 agosto.

Del Noce, A. (1978c). "Maritain: grandezza e limiti di un 'solitario”. Il Tempo, 18 novembre.

Del Noce, A. (1983a). "La lezione di Maritain”. En AA.VV. Atti del convegno di studi su Jacques Maritain. Napoli: Laurenziana, pp. 11-20. 
Del Noce, A. (1983b). "I nuovi e i vecchi zar al capolinea dell'ateismo". Il Sabato, VI, n. 25, 18 giugno.

Del Noce, A. (1983c). "Sette tesi quasi un epitaffio". 30 Giorni, I, n. 7, luglio.

Del Noce, A. (1985). "Intensità d'una grande illusione". Il Sabato, VIII, n. 17, 27 aprile.

Del Noce, A. (1986a). "L'umanesimo frainteso". 30 Giorni, IV, n. 4, aprile.

Del Noce, A. (1986b). "L'ora di una nuova laicità". Il Sabato, IX, n. 43, 25 ottobre.

Del Noce, A. (1986c). "Chi non si ferma al disincanto”. Il Sabato, IX, n. 48, 29 novembre.

Del Noce, A. (1987a). "Perché la fede salva la ragione". 30 Giorni, V, n. 7, luglio, pp. 44-47.

Del Noce, A. (1987b). "La morale cattocomunista". Il Sabato, X, n. 34, 22 agosto.

Del Noce, A. (1987c). "Soffocare tra le verità impazzite". Il Sabato, X, n. 38, 19 settembre.

Del Noce, A. (1988a). "Maritain messo in congedo". Il Tempo, 8 gennaio 1988.

Del Noce, A. (1988b). "Il Padrone del mondo”. 30 Giorni, VI, n. l, gennaio.

Del Noce, A. (1988c). "Così nacque il regime postfascista". Il Sabato, XI, n. 2, 9 gennaio.

Del Noce, A. (1988d). "Rivoluzione e Provvidenza”. Il Sabato, XI, n, 5, 30 gennaio.

Del Noce, A. (1988e). "I pericoli di un aggiornamento a rovescio". Il Tempo, 10 febbraio.

Del Noce, A. (1988f). "Una colomba, non un santo". Il Sabato, XI, n. 8, 20 febbraio.

Del Noce, A. (1988g). "Ascoltiamo quell'uomo". Il Sabato, a. X, n. 5, 1 febbraio.

Del Noce, A. (1988h). "Storia del cattolico borghese". Il Sabato, XI, n. 19, 7 maggio.

Del Noce, A. (1988i). "Cl, il Psi e il 'piano Scalfari”". 30 Giorni, VI, n. 10, ottobre.

Del Noce, A. (1988j). "La super ideologia”. Il Sabato, XI, n. 48, 26 novembre.

Del Noce, A. (1990). "L'inevitabile decomposizione del marxismo". 30 Giorni, VIII, n. 2, febbraio.

Del Noce, A. (1998). Cristianità e laicità. Scritti sul Il Sabato e latri anche inediti [CL], postfazione di Armellini, P. Milano: Giuffrè.

Del Noce, A. (2011) [1964]. Il problema dell'ateismo. Introduzione di N. Matteucci, postfazione d M. Cacciari. Bologna: Il Mulino.

Maritain, J. (1969) [1936]. Umanesimo integrale. Torino: Borla.

Mondin, B. (1990). "Attualità storica e riflessione metafisica". Prospettive nel mondo, XV, n. 163-164, gennaio-febbraio, pp. 58-62.

Napoleoni, C. (1990). Cercate ancora. Lettere sulla laicità e altri scritti. Roma: Editori Riuniti. 
Napoleoni, C. (1992). Dalla scienza all' utopia, a cura di G. L. Vaccarino. Torino: Bollati-Boringhieri.

Possenti, V. (1992), “Modernidad y Metafísica en A. Del Noce”. En Castellano, D. (ed.). Augusto Del Noce. Il pensiero filosófico. Nápoles: ESI.

Ricoeur, P. (1993). Sé stesso come un altro. Introducción y tr. it. de D. Jannotta. Milano: Jaca Book.

Tamassia, F. (1992). "Augusto Del Noce e l'analisi storica: il problema del Risorgimento”. En Castellano, D. (ed.). Augusto Del Noce. Il pensiero filosofico. Nápoles: ESI.

Vasale, C. (1995). "Etica e politica in Augusto Del Noce”. En AA.VV. Augusto Del Noce. Il problema della modernità. Roma: Studium. 